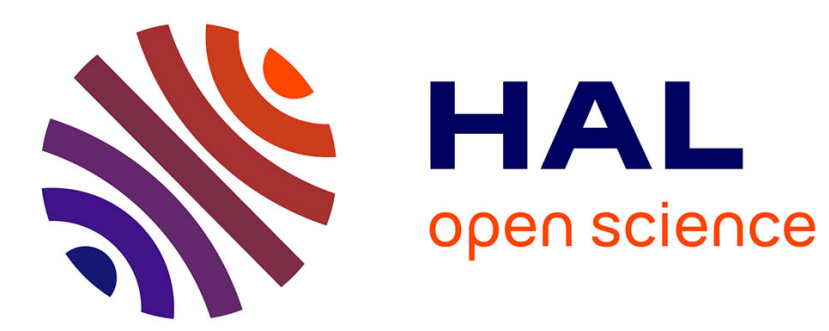

\title{
Action Principle for Non-Abelian Twisted Self-Duality Henning Samtleben
}

\section{To cite this version:}

Henning Samtleben. Action Principle for Non-Abelian Twisted Self-Duality. 2012. ensl-00679791

\section{HAL Id: ensl-00679791 https://hal-ens-lyon.archives-ouvertes.fr/ensl-00679791}

Preprint submitted on 16 Mar 2012

HAL is a multi-disciplinary open access archive for the deposit and dissemination of scientific research documents, whether they are published or not. The documents may come from teaching and research institutions in France or abroad, or from public or private research centers.
L'archive ouverte pluridisciplinaire HAL, est destinée au dépôt et à la diffusion de documents scientifiques de niveau recherche, publiés ou non, émanant des établissements d'enseignement et de recherche français ou étrangers, des laboratoires publics ou privés. 


\title{
Action Principle for Non-Abelian Twisted Self-Duality ${ }^{1}$
}

\author{
Henning Samtleben \\ Université de Lyon, Laboratoire de Physique, UMR 5672, CNRS et ENS de Lyon, \\ 46 allée d'Italie, F-69364 Lyon CEDEX 07, France \\ Institut Universitaire de France
}

\begin{abstract}
The dynamics of abelian vector and antisymmetric tensor gauge fields can be described in terms of twisted self-duality equations. The latter play an important role in exposing the duality symmetries of the theory. I this contribution I review recent progress in the construction of action principles for these equations and in particular their non-abelian generalizations.
\end{abstract}

\section{Introduction}

The dynamics of abelian vector and antisymmetric tensor gauge fields can be described by standard second order field equations or alternatively in terms of first order so-called twisted self-duality equations. These equations take the schematic form

$$
\mathcal{F}=\Omega \mathcal{M}^{*} \mathcal{F}
$$

where $\mathcal{F}$ combines the abelian field strengths of the original $p$-forms and their magnetic duals, and $\Omega \mathcal{M}$ denotes the 'twist matrix' which squares to the same multiple of the identity as the Hodge star '*' on the associated field strengths. References $[1,2]$ have coined the term of 'twisted self-duality equations'. Acting with an exterior derivative onto the system (1.1) reproduces the standard second order field equations for the $p$-form fields. A generalized version of the system (1.1) still holds in the presence of scalar fields, fermions, and Chern-Simons-type couplings as they typically arise in

\footnotetext{
${ }^{1}$ To appear in the Proceedings of the XVII European Workshop on String Theory, Padua, 5-9 September 201
} 
supersymmetric theories. In particular, the twist matrix in the general case depends on the scalar fields of the theory. The system (1.1) has a particularly interesting structure when referring to abelian $p$-forms in $D=(2 p+2)$-dimensional space-time, in which case $\mathcal{F}$ and ${ }^{*} \mathcal{F}$ are of equal rank, and the twist matrix can have non-trivial diagonal entries. Casting the $p$-form dynamics into the form (1.1) allows to exhibit the duality symmetries of these theories which are realized by a linear action on $\mathcal{F}$, instead of their hidden appearance in the standard second order framework.

The first-order equations (1.1) do not integrate to a standard Lorentz-covariant action. However, Henneaux and Teitelboim [3] and later Schwarz and Sen [4] have shown that these equations can be derived from a first-order action principle that carries on equal footing the $p$-forms together with their dual fields and is manifestly duality invariant, see [5, 6] for earlier work. The price to pay for duality invariance is to abandon manifest general coordinate invariance of the action. Although not manifest, the latter may be restored with a non-standard realization of space-time diffeomorphisms on the gauge fields. Alternatively, these theories can be obtained as the gauge-fixed versions of non-polynomial Lagrangians with manifest space-time symmetry [7, 8]. Recent applications of such actions have led to a Lagrangian for $N=8$ supergravity that possesses full $E_{7(7)}$ invariance, allowing to examine the role of the full $E_{7(7)}$ in perturbative quantization of the theory $[9,10]$.

In this contribution, we will describe the non-abelian generalization of the equations (1.1) and the corresponding duality invariant actions which have recently be constructed in [11], drawing on earlier work on non-abelian $p$-form tensor hierarchies in arbitrary dimensions $[12,13,14]$. We focus on the example of vector fields in fourdimensional space-time.

\section{Non-abelian gauge theory and two-form poten- tials in four dimensions}

Let us first review the covariant description of non-abelian gauge theory in four spacetime dimensions as inspired by the construction of gauged supergravity theories [15]. Combining electric and magnetic vector fields into a single set of gauge fields $A_{\mu}{ }^{M}$, labeled by a sympletic index $M=1, \ldots, 2 n$, the proper definition of covariant nonabelian field strengths furthermore requires the introduction of two-form potentials $B_{\mu \nu \alpha}$ transforming in the adjoint representation of the global duality group $G_{\text {duality }}$. Explicitly, these field strengths are given by

$$
\mathcal{H}_{\mu \nu}{ }^{M} \equiv 2 \partial_{[\mu} A_{\nu]}{ }^{M}+g X_{N P}{ }^{M} A_{[\mu}{ }^{N} A_{\nu]}^{P}-\frac{1}{2} g \Theta^{M \alpha} B_{\mu \nu \alpha}
$$


with a Stückelberg-type coupling to the two-form potentials $B_{\mu \nu \alpha}$. Here, the 'structure constants' $X_{N P}{ }^{M}$ are given as

$$
X_{N P}^{M} \equiv \Theta_{N}^{\alpha}\left(t_{\alpha}\right)_{P}^{M}
$$

in terms of the embedding tensor $\Theta_{N}{ }^{\alpha}$ and the generators $t_{\alpha}$ of the global duality group $G_{\text {duality }}$ of the ungauged theory. Symplectic indices are raised and lowered with the antisymmetric symplectic tensor $\Omega^{M N}$. In four space-time dimensions, the embedding tensor $\Theta_{M}{ }^{\alpha}$ is constrained by the linear relations

$$
X_{(M N K)}=0
$$

and moreover is subject to the bilinear constraints

$$
\begin{aligned}
f_{\alpha \beta}^{\gamma} \Theta_{M}^{\alpha} \Theta_{N}^{\beta}+\left(t_{\alpha}\right)_{N}{ }^{P} \Theta_{M}{ }^{\alpha} \Theta_{P}^{\gamma} & =0, \\
\Omega^{M N} \Theta_{M}{ }^{\alpha} \Theta_{N}{ }^{\beta} & =0,
\end{aligned}
$$

of which the first corresponds to a generalized Jacobi identity and the second one insures locality of the gauging (i.e. the existence of a symplectic frame in which all magnetic charges vanish). Local gauge transformations are given by

$$
\begin{aligned}
\delta_{\Lambda} A_{\mu}{ }^{M} & =D_{\mu} \Lambda^{M}+\frac{1}{2} g \Theta^{M \alpha} \Lambda_{\mu \alpha} \\
\delta_{\Lambda} B_{\mu \nu \alpha} & =2 D_{[\mu} \Lambda_{\nu] \alpha}-2\left(t_{\alpha}\right)_{M N}\left(\Lambda^{M} \mathcal{H}_{\mu \nu}{ }^{N}-A_{[\mu}{ }^{M} \delta_{\Lambda} A_{\nu]}{ }^{N}\right)
\end{aligned}
$$

with parameters $\Lambda^{M}$ and $\Lambda_{\mu \alpha}$, and covariant derivatives defined according to

$$
D_{\mu} \equiv \partial_{\mu}-g A_{\mu}{ }^{M} X_{M} \equiv \partial_{\mu}-g A_{\mu}{ }^{M} \Theta_{M}{ }^{\alpha} t_{\alpha}
$$

Under these gauge transformations, the field strengths (2.1) transform covariantly as

$$
\delta_{\Lambda} \mathcal{H}_{\mu \nu}{ }^{M}=-g \Lambda^{K} X_{K N}{ }^{M} \mathcal{H}_{\mu \nu}{ }^{N}
$$

The presence of the two-form fields in (2.1) is crucial for the covariant transformation behavior of the non-abelian field strengths. We finally mention that the covariant field strengths (2.1) satisfy the generalized Bianchi identities

$$
D_{[\mu} \mathcal{H}_{\nu \rho]}^{M}=-\frac{1}{6} g \Theta^{M \alpha} \mathcal{H}_{\mu \nu \rho \alpha}
$$

with the covariant non-abelian field strength $\mathcal{H}_{\mu \nu \rho \alpha}$ of the two-form tensor fields, given by

$$
\Theta_{M}{ }^{\alpha} \mathcal{H}_{\mu \nu \rho \alpha}=3 \Theta_{M}^{\alpha} D_{[\mu} B_{\nu \rho] \alpha}+6 X_{M P Q} A_{[\mu}{ }^{P}\left(\partial_{\nu} A_{\rho]}{ }^{Q}+\frac{1}{3} g X_{R S}{ }^{Q} A_{\nu}{ }^{R} A_{\rho]}{ }^{S}\right)(2.1
$$




\section{Action for non-abelian twisted self-duality equa- tions}

Having defined non-abelian field strengths for the symplectically covariant vector $A_{\mu}{ }^{M}$, we can now formulate a duality covariant action principle by properly generalizing the construction of Henneaux and Teitelboim [3]. As a first step, we split the fourdimensional coordinates into $\left\{x^{\mu}\right\} \rightarrow\left\{x^{0}, x^{i}\right\}$ and the metric as

$$
g_{\mu \nu}=\left(\begin{array}{cc}
-N^{2}+h_{i j} N^{i} N^{j} & h_{i j} N^{i} \\
h_{i j} N^{j} & h_{i j}
\end{array}\right),
$$

into the standard lapse and shift functions. The full non-abelian Lagrangian then takes the form

$$
\begin{aligned}
\mathcal{L}_{\text {covariant }}= & \frac{e_{3}}{2 N} h^{i j}\left(\mathcal{E}_{i}{ }^{M}-\mathcal{B}_{i}{ }^{M}\right) \mathcal{M}_{M N} \mathcal{B}_{j}{ }^{N} \\
& +\frac{1}{16} \varepsilon^{\mu \nu \rho \sigma} g \Theta_{M}{ }^{\alpha} B_{\mu \nu \alpha} \mathcal{H}_{\rho \sigma}{ }^{M}+\frac{1}{12} \varepsilon^{\mu \nu \rho \sigma} g X_{P Q M} \partial_{\mu} A_{\nu}{ }^{M} A_{\rho}{ }^{P} A_{\sigma}{ }^{Q} \\
& +\mathcal{L}_{\text {matter }}
\end{aligned}
$$

with the covariant electric and magnetic fields $\mathcal{E}_{i}{ }^{M}, \mathcal{B}_{i}{ }^{M}$ given by

$$
\begin{aligned}
\mathcal{E}_{i}{ }^{M} & \equiv \mathcal{H}_{0 i}{ }^{M}-N^{j} \mathcal{H}_{j i}{ }^{M}, \\
\mathcal{B}_{i}{ }^{M} & \equiv-\frac{1}{2} e_{3} \varepsilon_{i j k} N \Omega^{M N} \mathcal{M}_{N K} \mathcal{H}^{j k K}
\end{aligned}
$$

in terms of the non-abelian field strengths from (2.1). The first line of (3.1) is the straight-forward covariantization of $[3,4]$, the second line is a new non-abelian gaugeinvariant topological term, required in order to reproduce the correct field equations. Finally, $\mathcal{L}_{\text {matter }}$ comprises possible matter couplings (to scalars, fermions, gravity). Formally, the Lagrangian (3.1) is invariant under the full action of the global duality group $G_{\text {duality }}$, if the embedding tensor $\Theta_{M}{ }^{\alpha}$ is treated as a spurionic object transforming under $G_{\text {duality }}$. A concrete choice of the embedding tensor will specify a particular gauging and explicitly break the duality group. After some work it can be shown, that the Lagrangian (3.1) implies the following set of equations of motion

$$
\begin{aligned}
\mathcal{E}_{i}{ }^{M} & =\mathcal{B}_{i}{ }^{M} \\
\Theta_{M}{ }^{\alpha} \mathcal{H}_{\mu \nu \rho \alpha} & =-2 e_{4} \varepsilon_{\mu \nu \rho \sigma} j^{\sigma}{ }_{M}, \quad \text { with } \quad j^{\sigma}{ }_{M} \equiv e_{4}^{-1} g^{-1} \frac{\partial \mathcal{L}_{\text {matter }}}{\partial A_{\sigma}{ }^{M}}
\end{aligned}
$$

The first of these equations is a rewriting of the four-dimensional twisted self-duality equation (1.1)

$$
\mathcal{H}_{\mu \nu}{ }^{M}=-\frac{1}{2} e_{4} \varepsilon_{\mu \nu \rho \sigma} \Omega^{M N} \mathcal{M}_{N K}\left(\phi^{i}\right) \mathcal{H}^{\rho \sigma K}
$$


while the second equation is the four-dimensional duality equation relating the two-form fields to the scalar fields or, more precisely, to the Noether current of the invariances that have been gauged. It shows that the two-form gauge potentials, introduced in the process of gauging the theory, do not constitute new degrees of freedom but are in fact non-propagating fields (or the on-shell duals to the scalar fields of the theory). In the abelian limit, these equations consistently decouple form the theory. The set of equations (3.4) precisely parallels the equations of motion obtained from the secondorder formalism with magnetic gauge fields of [15].

Let us finally describe the symmetries of the Lagrangian (3.1). The key structure is its general variation, that can be expressed as

$$
\begin{aligned}
\delta \mathcal{L}_{\text {covariant }}= & \varepsilon^{i m n}\left\{\Omega_{M N} D_{m}\left(\mathcal{E}_{n}{ }^{N}-\mathcal{B}_{n}{ }^{N}\right)+\frac{1}{4} g \Theta_{M}{ }^{\alpha} \mathcal{H}_{0 m n \alpha}\right\} \delta A_{i}{ }^{M} \\
& -\frac{1}{4} \varepsilon^{i m n} g \Theta_{M}{ }^{\alpha}\left(\mathcal{E}_{i}{ }^{M}-\mathcal{B}_{i}{ }^{M}\right)\left(\delta B_{m n \alpha}-2\left(t_{\alpha}\right)_{K L} A_{[m}{ }^{K} \delta A_{n]}{ }^{L}\right) \\
& -\frac{1}{12} \varepsilon^{i m n} g \Theta_{M}{ }^{\alpha} \mathcal{H}_{\text {imn } \alpha} \delta A_{0}{ }^{M}+\delta \mathcal{L}_{\text {matter }} .
\end{aligned}
$$

Based on this variation, the Lagrangian can be shown to be invariant under the local gauge transformations (2.6). Although four-dimensional coordinate invariance is not manifest, it follows from (3.6) after some calculation, that the Lagrangian is also invariant under time-like diffeomorphisms $\xi^{0}$, provided the gauge field potentials transform as

$$
\begin{aligned}
\delta A_{i}{ }^{M} & =\xi^{0}\left(\mathcal{B}_{i}{ }^{M}+N^{j} \mathcal{H}_{j i}{ }^{M}\right), \\
\delta A_{0}{ }^{M} & =0 \\
\Theta_{M}{ }^{\alpha} \delta B_{i j \alpha} & =-2 e_{3} N \xi^{0} \varepsilon_{i j k} j^{k}{ }_{M}+2 X_{M K L} A_{[i}{ }^{K} \delta A_{j]}{ }^{L},
\end{aligned}
$$

with the spatial component of the current $j^{k}{ }_{M}$ from (3.4). On-shell, i.e. upon using the equations of motion (3.3), (3.4), these transformation laws reduce to

$$
\begin{aligned}
\delta A_{i}{ }^{M} & \approx \xi^{0} \mathcal{H}_{0 i}{ }^{M} \\
\Theta_{M}{ }^{\alpha} \delta B_{i j \alpha} & \approx \Theta_{M}{ }^{\alpha}\left(\xi^{0} \mathcal{H}_{0 i j \alpha}+2\left(t_{\alpha}\right)_{P Q} A_{[i}{ }^{P} \delta A_{j]}{ }^{Q}\right)
\end{aligned}
$$

which in turn reproduce the standard transformation behavior under time-like diffeomorphisms up to local gauge transformations (2.6) with parameters $\Lambda^{M} \equiv-\xi^{0} A_{0}{ }^{M}$, $\Lambda_{\mu \alpha} \equiv-B_{\mu i \alpha}-\left(t_{\alpha}\right)_{K L} A_{\mu}{ }^{K} A_{0}{ }^{L}$. In the abelian case, the modified form of time-like diffeomorphisms (3.7) for the vector fields $A_{i}{ }^{M}$ coincides with the results of $[3,4]$. The main difference in the non-abelian case (apart from standard gauge covariantization) is the explicit appearance of the gauge fields in the matter part $\mathcal{L}_{\text {matter }}$ of the Lagrangian. Since these couplings are manifestly four-dimensional coordinate covariant, the off-shell modification of the transformation law (3.7) leads to extra contributions 
from this sector which are proportional to the equations of motion

$$
\delta_{\text {extra }} \mathcal{L}=\frac{\partial \mathcal{L}_{\text {matter }}}{\partial A_{i}{ }^{M}} \xi^{0}\left(\mathcal{B}_{i}{ }^{M}-\mathcal{E}_{i}{ }^{M}\right)
$$

With (3.6) and (3.4), these contributions are precisely cancelled by the modified transformation law of $B_{i j}{ }^{M}$ in (3.7). To summarize, the duality covariant Lagrangian (3.1) is invariant under local gauge transformations (2.6) and also under four-dimensional diffeomorphisms with the modified transformation laws (3.7).

This concludes the non-abelian generalization of the Henneaux-Teitelboim action principles. Among other applications, it would be highly interesting to generalize the presented construction to the non-polynomial Lagrangians with manifest space-time symmetry $[7,8]$ from which the first-order actions $[3,4]$ are obtained after particular gauge fixing.

\section{Acknowledgements}

I thank the organizers for a great and inspiring meeting.

\section{References}

[1] E. Cremmer, B. Julia, H. Lu, and C. N. Pope, Nucl. Phys. B523, 73-144 (1998).

[2] E. Cremmer, B. Julia, H. Lu, and C. N. Pope, Nucl. Phys. B535, 242-292 (1998).

[3] M. Henneaux and C. Teitelboim, Phys. Lett. B206, 650 (1988).

[4] J. H. Schwarz and A. Sen, Nucl. Phys. B411, 35-63 (1994).

[5] S. Deser and C. Teitelboim, Phys. Rev. D13, 1592-1597 (1976).

[6] R. Floreanini and R. Jackiw, Phys. Rev. Lett. 59, 1873 (1987).

[7] P. Pasti, D. P. Sorokin, and M. Tonin, Phys. Rev. D52, 4277-4281 (1995).

[8] P. Pasti, D. P. Sorokin, and M. Tonin, Phys. Rev. D55, 6292-6298 (1997).

[9] C. Hillmann, JHEP 04, 010 (2010).

[10] G. Bossard, C. Hillmann, and H. Nicolai, JHEP 12, 052 (2010).

[11] H. Samtleben, Nucl. Phys. B851, 298-313 (2011).

[12] B. de Wit and H. Samtleben, Fortschr. Phys. 53, 442-449 (2005).

[13] B. de Wit, H. Nicolai, and H. Samtleben, JHEP 02, 044 (2008).

[14] E. A. Bergshoeff, J. Hartong, O. Hohm, M. Hübscher, and T. Ortin, JHEP 04, 123 (2009).

[15] B. de Wit, H. Samtleben, and M. Trigiante, JHEP 09, 016 (2005). 